\title{
INCREASING FLUX DENSITY BY HTS SHIELDING PELLET IN SUPERCONDUCTING SYNCHRONOUS MACHINE BASED ON FLUX CONCENTRATION
}

\author{
R. BOUMARAF", S. M. MIMOUNE ${ }^{* *}$ L. ALLOUI", M. L. KHENE \\ "Laboratoire de Génie Electrique de Biskra LGEB, Université de Biskra, BP 145, Biskra, Algérie \\ "Laboratoire de Modélisation des Systèmes Energétiques LMSE, Université de Biskra, BP 145, Biskra, Algérie \\ r.boumaraf@univ-biskra.dz
}

\begin{abstract}
In this paper, we propose a solution which enhances the performance of the inductor in high-power superconducting synchronous machines based on the flux concentration. The work is done by keeping the same topology and using a high temperature superconducting shielding pellet located between the two coils of the inductor. This pellet permits to recover the magnetic field which vanishes in the medium region due to the opposite direction of the coils. We did a 3D magnetostatic field analysis using the control volume method with unstructured grid. This analysis showed that the suggested solution allowed obtaining a maximum efficiency of about $8 \%$ in the flux density.
\end{abstract}

INDEX TERMS: Control volume method, Flux concentration, HTS shielding pellet, Superconducting synchronous machines

\section{INTRODUCTION}

High-temperature superconductors (HTS) have created opportunities for a great leap forward in the technology of large electrical machines. HTS motors and generators will operate as conventional machines and became smaller, lighter, more efficient, and less expensive to manufacture [1]. Most of classical superconducting motors have been designed for marine propulsion and wind power system, with a classical geometry using superconducting inductor with the same cryogenic conditions [2]. Complex topologies have been suggested for superconducting lowpower motors based on axial high flux density and complex design as for general aviation aircraft [3].

High-power HTS synchronous machines based on the flux concentration have been developed and manufactured. These machines have shown successful results on the load test [4]. In fact, the previous superconducting inductor has been optimized and its new geometry provides higher performance when using NbTi wires [5]. In the same way, a modification of HTS shield length should decrease the magnetic flux concentration.

To increase the power in these types of machines, several ways are proposed. The first obvious solution suggests new superconducting wires with higher current density possibility, the second one consists in a biggest inductor radius dimension and the third alternative suggests exploring a long inductor length [6].

In this work, we propose an ingenious solution by using a HTS shielding pellet between the two coils of the inductor. The introduction of this pellet enhances the performance of the inductor while keeping the same geometry as in the previous topology. The inserting of the pellet has the goal to recover the magnetic field that vanishes in the medium region due to the opposite direction of coils in previous geometry. To model the magnetostatic field, we adopted in this work a three dimensional (3D) magnetostatic field analysis using the control volume method (CVM) with unstructured grid.

\section{PROPOSED DESIGN}

The previous structure of the superconducting inductor is based on the flux concentration (Fig. 1). The inductor uses both low-temperature superconductors (LTS) NbTi wires and High temperature superconductors (HTS) YBCO bulk. The LTS NbTi wires create a high magnetic field through two coaxial coils fed by opposite currents. Four YBCO bulk plates are located between the two coils to screen the normal component of the magnetic field. They are used as magnetic shields, shape the flux lines and then provide a spatial magnetic field variation.

The inductor configuration allows a good flux concentration between the four YBCO shielding bulk plates and increases the magnetic flux density in the air gap providing a high power density and a high electromagnetic torque. The inductor is kept stationary in order to simplify the cryogenics and minimize cold losses [5].

To increase moreover the power density of the superconducting motor by increasing magnetic flux density several ways are possible. One solution suggests new 
superconducting wires with higher current density possibility but this solution is limited by a maximum current density. Second one consists in a biggest inductor radius dimension, but a limit appears with the superconducting wire length. Third one is to explore a long inductor length but this modification of HTS shield length should decrease the magnetic flux concentration [6].

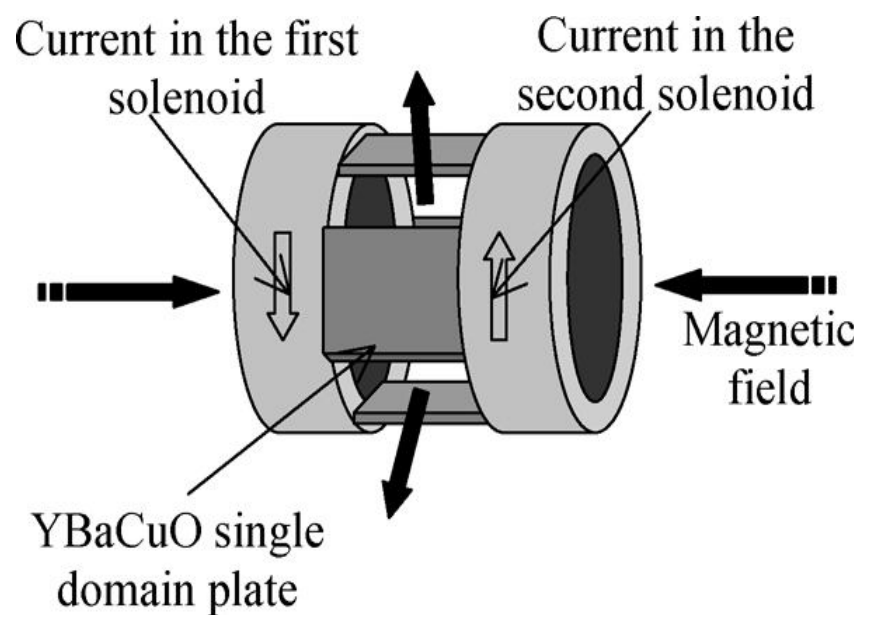

Figure 1: Topology of the previous inductor [4].

In fact, the previous superconducting inductor has been optimized and this geometry provides the best performance with $\mathrm{NbTi}$ wires. Moreover, we can suggest a new inductor topology with the same LTS solenoids and HTS bulks to increase the power while keeping the same geometry. The ingenious solution is done by using a HTS YBCO shielding pellet located between the two coils (Fig. 2). The pellet has a cylindrical form with optimized dimensions.

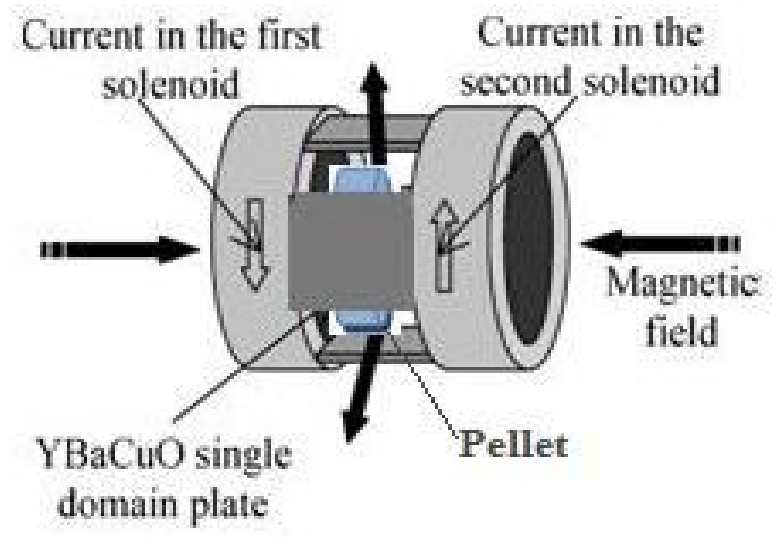

Figure 2: Topology of the proposed inductor.

This pellet allows recovering the magnetic field which vanishes in the medium region due to the opposite direction of coils (Fig. 3). A field calculation shows the feasibility of this structure. The shielding pellet deflects the field lines lost and then increases the radial magnetic flux density in the air-gap of the machine.
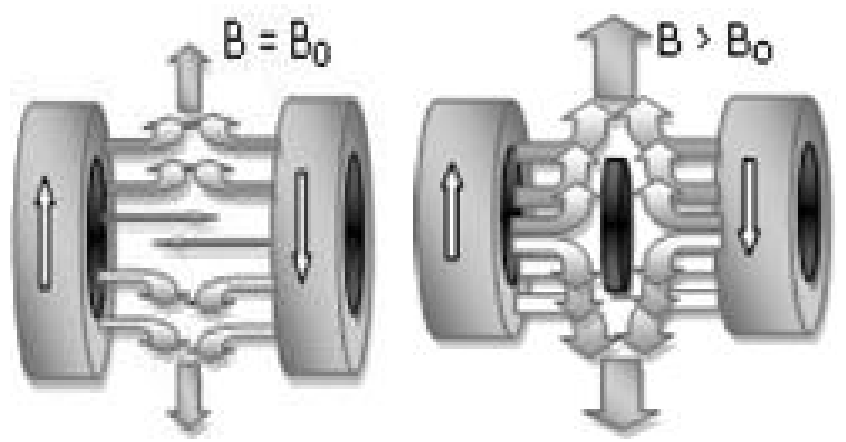

Figure 3: Pellet effect on increasing magnetic flux density.

\section{MAGNETIC MODEL}

To calculate the magnetic flux density in the design, we calculate the magnetic scalar potential $\mathrm{V}$ from using the Coulomb model of coils [5]. The calculation of the gradient of the potential gives the magnetic field. Superconducting material is assumed to be perfectly diamagnetic and therefore Neumann conditions are considered at the boundaries. Figure 4 shows the inductor dimensions.

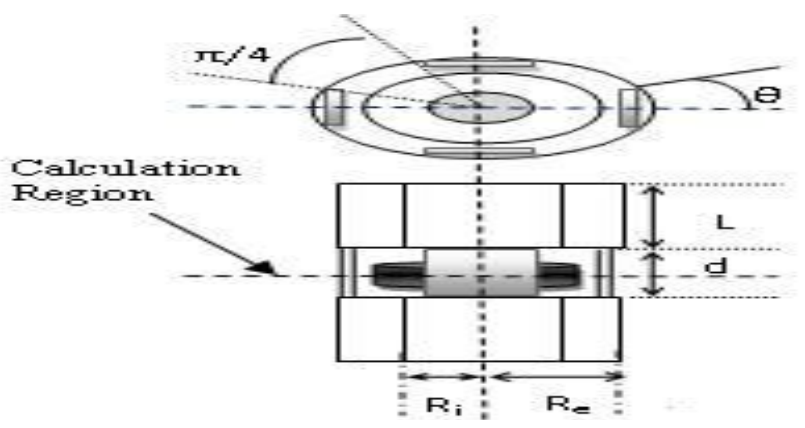

Figure 4: Inductor dimensions: $R i=23 \mathrm{~mm}, R e=33 \mathrm{~mm}, d=25$ $\mathrm{mm}, L=45 \mathrm{~mm}$.

The equations system is as follow:

$$
\left\{\begin{array}{l}
\Delta V=0 \\
\left.\frac{\partial V}{\partial z}\right|_{1}-\left.\frac{\partial V}{\partial z}\right|_{2}= \pm \sigma \\
\frac{\partial V}{\partial z}=0 \text { on the surface of sup raconductor } \\
V=0 \text { on the boundary }
\end{array}\right.
$$

The Coulomb model of coils as presented in [4] is:

$$
\left\{\begin{array}{lll}
\sigma_{m 1}=\mu_{0} J_{s}\left(R_{e}-R_{i}\right) & \text { if } & 0<r<R_{i} \\
\sigma_{m 2}=\mu_{0} J_{s}\left(R_{e}-r\right) & \text { if } & R_{i}<r<R_{e}
\end{array}\right.
$$


In order to show the effectiveness of the pellet on increasing the flux density, we have developed a 3D numerical code based on the control volume method CVM dedicated to modeling electromagnetic phenomena in devices with complex geometries.

\section{CALCULATION METHOD}

The CVM can be seen as a special version of the weighted residuals method. It is one of the numerical methods widely used in computation of heat and fluid dynamic problems [7]. Furthermore, this method has been applied successfully to a variety of electromagnetic problems [8-11]. It uses a support space mesh based on an unstructured grid (Fig. 5 left) and it consists in dividing the studied domain into a number of $3 \mathrm{D}$ control volumes scheme surrounding each node (Fig. 5 right).

The scheme of the 3D control volume $\mathrm{Dp}$ is displayed in (Fig. 5 right) and is limited by several facets related to neighboring nodes of the principal node $\mathrm{P}$.

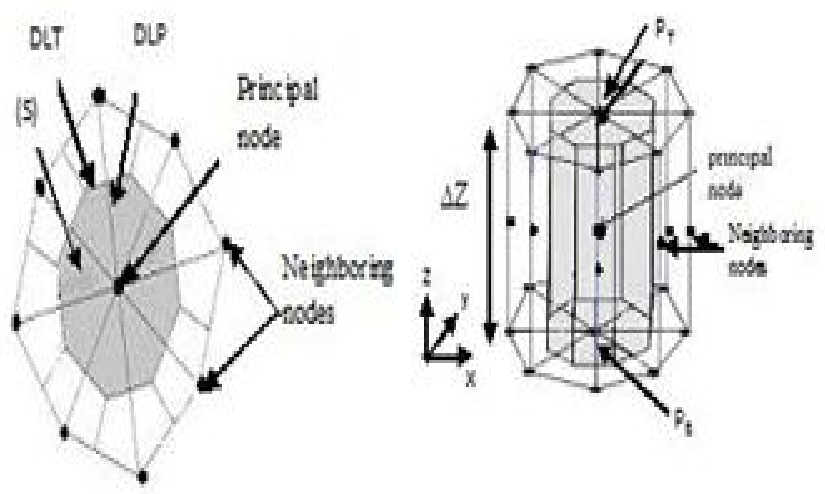

Figure 5: 2D support mesh (left) and 3D control volume scheme (right).

The Laplacian in (1) is integrated over each $3 \mathrm{D}$ control volume $\mathrm{D}_{\mathrm{p}}$ as follow:

$\iiint_{D_{p}} \nabla \cdot(\nabla V) d \tau=0$

By using the theorem of Ostrogradski, the divergence volume integrals of (3) are transformed into surface integrals:

$$
\iiint_{\int_{p}} \nabla \cdot(\nabla V) d \tau=\iint_{\sum_{s i} l_{a i}} \nabla V d s l_{a i}+\iint_{k=T: B} \nabla V d s_{k}
$$

The right hand side terms in Eq. (4) represents the flux of the magnetic potential $\mathrm{V}$ through the lateral facets and the top and bottom facets respectively.

\section{ADOPTED APPROXIMATION}

To calculate top and bottom terms, a first order approximation is used.

As the flux in the top facet is:

$$
\iint \nabla V d s_{T}=\frac{V^{T}-V^{p}}{\Delta z_{t}} \Delta s_{t}
$$

To calculate the flux in the lateral facets, the magnetic scalar potential V is expressed in referential (R') defined by $(\xi, \eta)$ coordinates following the same procedure as in [11]. Using this transformation, we can express the derivative of the magnetic scalar potential in the referential (R).

So, the flux in the lateral facets can be expressed as:

$$
\begin{aligned}
& \iint_{\sum_{d s l_{a i}}} \nabla V d s l_{a i}=\sum_{\substack{i=l . \eta E E i \\
R=\eta, \zeta}} a_{R E i} V_{R E i}-a p V_{p} \\
& \text { Where : } \quad a_{R E i}=a x_{R E i}+a y_{R E i} \\
& a p=\sum_{R=\eta, \zeta} a_{R E i}
\end{aligned}
$$

The coefficients $a_{R E i}$ describe the physical and geometrical properties of the control volume. An algebraic system of equations is constructed with the appropriate boundary conditions of the computational domain as in (1). The solution is obtained by solving iteratively the algebraic system using the Gauss Seidel method when convergence is reached.

\section{RESULTS}

The performance of the new proposed topology of the inductor is conducted by varying the dimensions of the cylindrical pellet. The three-dimensional analysis based on the control volume method allows a complete magnetic flux density map on each point of the mesh.

To validate the numerical model, we used the experimental data of the previous topology of the inductor (without pellet) giving the magnetic flux density versus the angular position numbered from S1 to S10 [5].

Figure 6 presents a comparison between the measured flux densities and the calculated ones for inductor's radius 33 $\mathrm{mm}$ and coils current 260A. A good agreement is obtained for the angular positions S1, S5, S7 and S9. The difference between the calculated and measured [5] flux density for 
the angular positions $\mathrm{S} 2, \mathrm{~S} 3, \mathrm{~S} 4, \mathrm{~S} 6, \mathrm{~S} 8$ and $\mathrm{S} 10$ is due that the measured positions which are lower than the calculated radius $33 \mathrm{~mm}$.
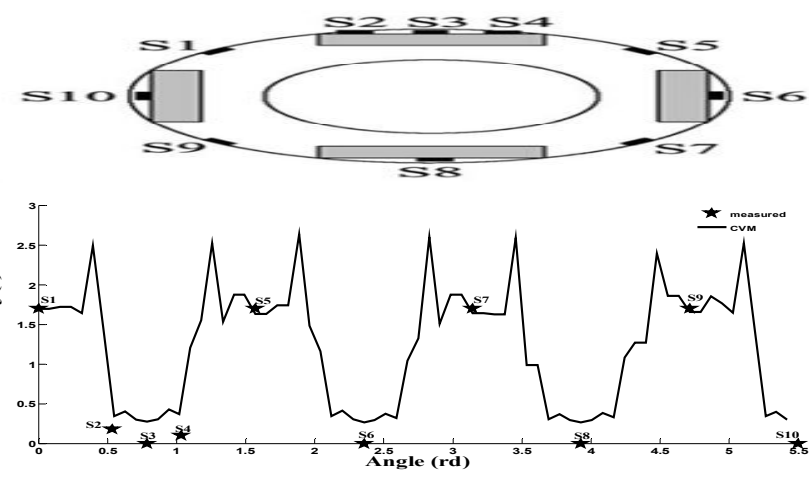

Figure 6: Comparison of measured [5] and calculated magnetic flux densities of the previous topology inductor (for radius equal to $33 \mathrm{~mm}$ ) (l=260A).

Figure 7 shows the distribution of the magnetic scalar potential and the vector magnetic flux density in the medium plane of the inductor in the case of the inductor without pellet.
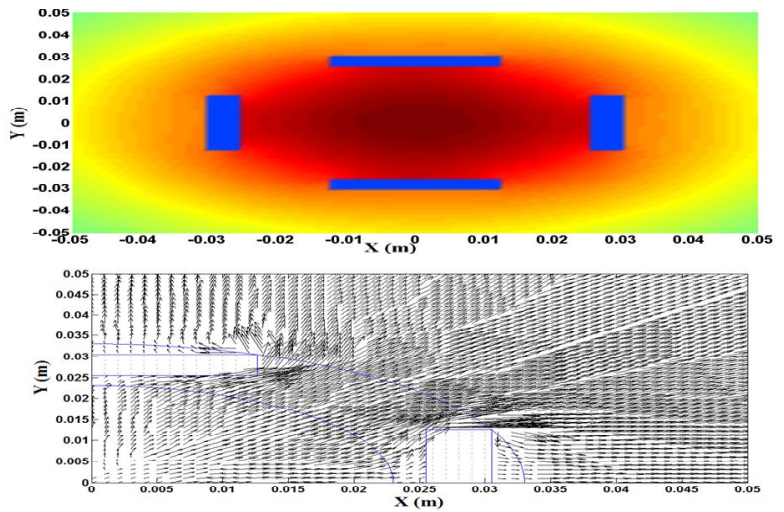

Figure 7: Distribution of the magnetic scalar potential (left) and the magnetic flux density (right) in the medium plane of the inductor without pellet.

In order to optimize the shielding pellet dimensions to have In order to optimize the shielding pellet dimensions to have a maximum flux density in the air-gap of the machine and between each pair of the shielding bulks, we introduced several dimensions of the pellet.

There are two mainly parameters to optimize the shielding pellet: the radius $\mathrm{R}$ and the thickness $\mathrm{E}$. We define as example "R5E13" the pellet sample of radius $\mathrm{R}$ equal to 5 $\mathrm{mm}$ and thickness E equal to $13 \mathrm{~mm}$.

We evaluated for several pellet samples, the magnetic flux density in the contour line of inductor's radius forming the air-gap of the machine and equal to $33 \mathrm{~mm}$. Data are presented in table 1.

Table 1 shows the magnetic flux density efficiency (relative error) of the topology with pellet compared to the topology without pellet for various samples in the angular position S5.

Table I: Magnetic flux density efficiency using HTS pellet

\begin{tabular}{cccccc}
\hline $\mathrm{E} / \mathrm{R}$ & $5 \mathrm{~mm}$ & $10 \mathrm{~mm}$ & $13 \mathrm{~mm}$ & $16 \mathrm{~mm}$ & $19 \mathrm{~mm}$ \\
\hline $5 \mathrm{~mm}$ & $3.92 \%$ & $4.59 \%$ & $4.82 \%$ & $8.12 \%$ & $-2.46 \%$ \\
\hline $10 \mathrm{~mm}$ & $3.65 \%$ & $4.54 \%$ & $4.80 \%$ & $7.58 \%$ & $-3.53 \%$ \\
\hline
\end{tabular}

Calculations show that for the two thicknesses, the magnetic flux density increases with the pellet's radius and maximums are reached for radius equal to $16 \mathrm{~mm}$. At this radius, the maximum efficiency is of about $8 \%$ for the optimized sample E5R16. When the pellet's radius exceeds the $16 \mathrm{~mm}$, the pellet approaches the air-gap of $33 \mathrm{~mm}$ and it deviates moreover the magnetic flux density from the middle plane. In this case the magnetic flux density increases faraway a middle plane and the HTS pellet has a negative effect.

Figure 8 shows the magnetic flux density for the optimized sample compared to the case without pellet. Figure 9 and 10 , show respectively the magnetic scalar potential and the magnetic flux density along a shielding bulk plate at angle $\theta$ equal to zero degree (Fig. 4).

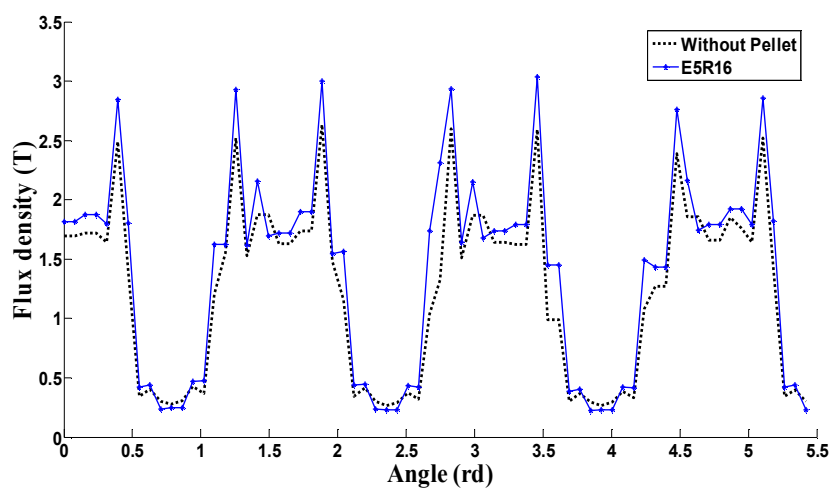

Figure 8: Flux density in the contour line of the air-gap of radius equal to $33 \mathrm{~mm}$ for optimized sample E5R16

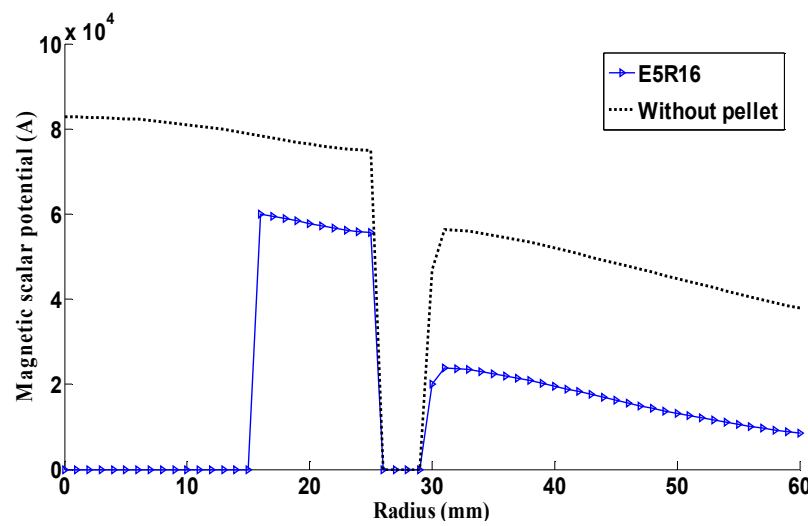

Figure 9: Magnetic scalar potential along a shielding bulk Plate for optimized sample E5R16 and for without pellet $(\theta$ equal to zero degree). 


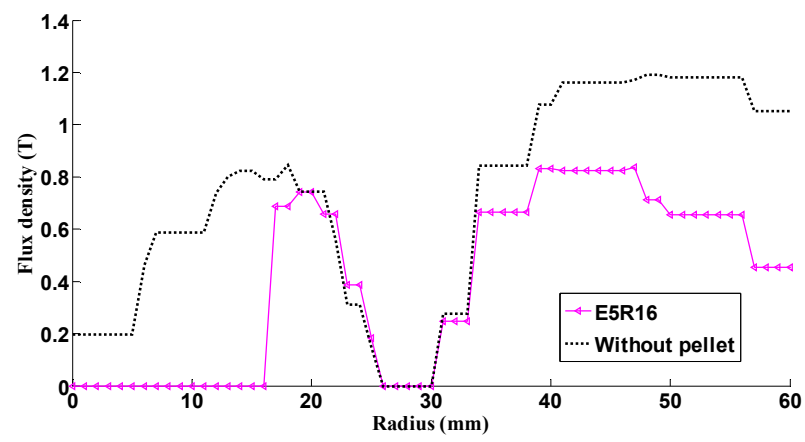

Figure 10: Magnetic flux density along a shielding bulk Plate for optimized sample E5R16 and for without pellet $(\theta$ equal to zero degree).

Figure 11 and 12, show respectively the magnetic scalar potential and the magnetic flux density between two shielding bulk plates at angle $\theta$ equal to 45 degree. Figure 12 shows that in the presence of the pellet (E5R16), the value of the magnetic flux density of the inductor increases near the air-gap S5.

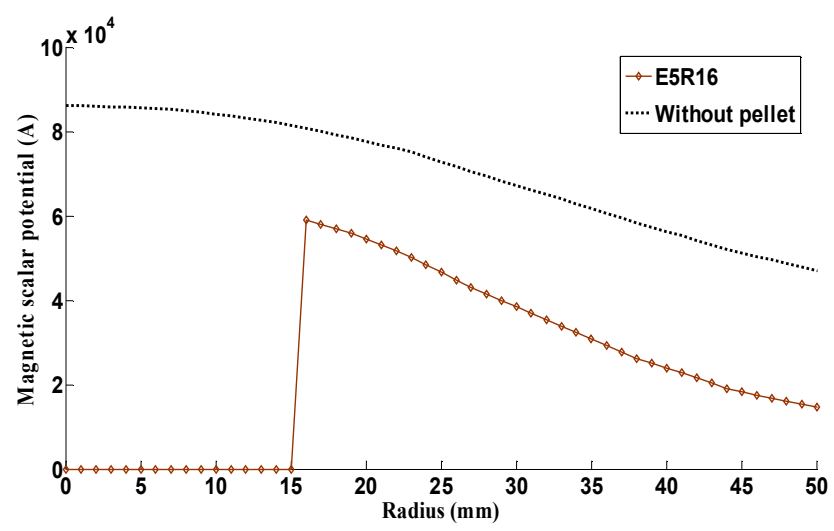

Figure 11: Magnetic scalar potential between tow shielding bulk Plates for optimized sample E5R16 and for without pellet ( $\theta$ equal to 45 degree).

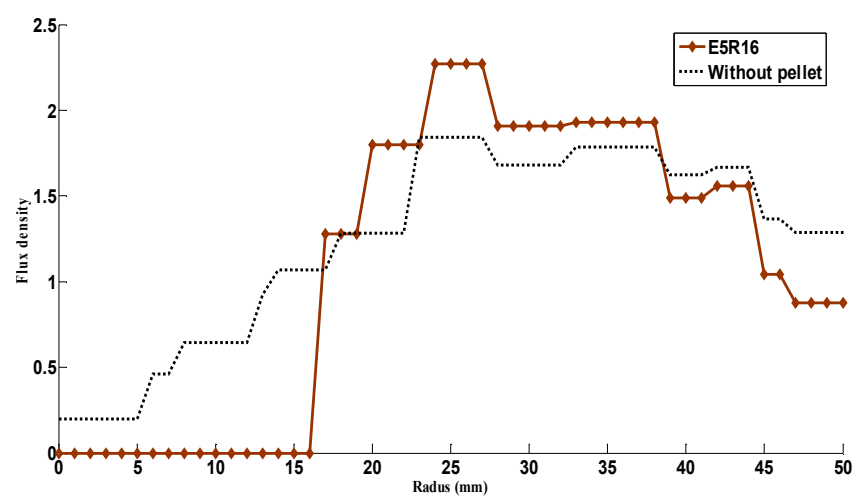

Figure 12: Magnetic flux density between tow shielding bulk Plates for optimized sample E5R16 and for without pellet ( $\theta$ equal to 45 degree).

\section{CONCLUSION}

In this paper, we proposed a solution which enhances the performance of the superconducting inductor in superconducting synchronous machine based on flux concentration by using HTS shielding pellet. The numerical calculation is performed by the control volume method and the comparison of the numerical code results with experiments verifies the validity of the numerical model for the previous inductor topology. The new configuration of the inductor permits to obtain a maximum efficiency of the magnetic flux density in the air-gap of about $8 \%$ for pellet sample of radius $16 \mathrm{~mm}$ and thickness $5 \mathrm{~mm}$. When the pellet's radius comes near the air-gap, the rise of the magnetic flux density is not significant and the HTS pellet has a negative effect.

\section{REFERENCES}

[1] Swarn S. Kalsi "Development Status of Superconducting Rotating Machines," IEEE PES Meeting, New York, 27-31 January 2002

[2] P. Tixador, F. Simon, H. Daffix, and M. Deleglise, "150-kW experimental superconducting permanentmagnet motor," IEEE Trans. Appl. Supercond., vol. 9, no. 2, pp. 1205-1208, Jun. 1999.

[3] P. J. Masson, P. Tixador, and C. A. Luongo, "Safety torque generation in HTS propulsion motor for general aviation aircraft," IEEE Trans. Appl. Supercond,, vol. 17, no. 2, pp. 1619-1622, Jun. 2007.

[4] E. Ailam, D. Netter, J. Lévêque, B. Douine, P. Masson, and A. Rezzoug, "Design and testing of a superconducting motor," IEEE Trans. Appl. Supercond.., vol. 17, no. 1, pp. 27-33, Mar. 2007.

[5] P. Masson, D. Netter, D. Leveque, and A. Rezzoug, "Experimental study of a new kind of superconducting inductor," IEEE Trans. Appl.Supercond., vol. 13, no. 2, pp. 2239-2242, Jun. 2003.

[6] R.Moulin, J. Lévêque, L. Durantay, B. Douine, D. Netter, and A. Rezzoug, " Superconducting Multistack Inductor for Synchronous Motors Using the Diamagnetism Property of Bulk Material," IEEE Trans. Ind. Electronics., vol. 57, no. 1, pp. 146-153, Jun. 2010.

[7] S. V. Patankar, "Numerical heat transfer and fluid flow", Series in computational methods in mechanics and thermal sciences. Hemisphere publishing corporation, 1980.

[8] A. Cheriet, M. Feliachi, S. M. Mimoune, "Nonconforming mesh generation for finite volume method applied to 3-D magnetic field analysis", The European Physical Journal Applied Physics EPJAP, Vol. 37, issue 2, pp. 191-195, February 2007.

[9] A. Cheriet, M. Feliachi, S. M. Mimoune, "3-D movement simulation technique in FVM method application to eddy current non-destructive testing", The International Journal for Computation and Mathematics in Electrical and Electronic Engineering COMPEL, Vol. 28, issue 1, pp. 77-84, January 2009. 
[10] L. Alloui, F. Bouillault, S. M. Mimoune, "Numerical study of the influence of flux creep and of thermal effect on dynamic behaviour of magnetic levitation systems with a high- $T c$ superconductor using control volume method" EPJ. App. Phys. 37 (2) (2009) 191.
[11] L. Alloui, F. Bouillault, L. Bernard, J. Lévêque, and S. M. Mimoune,"3D modeling of forces between magnet and HTS in a levitation system using new approach of the control volume method based on anunstructured grid," Physica C 475, pp 32-37, 2012. 